\title{
SUPPLY CHAIN MODELING WITH UNCERTAINTY IN DEMAND AND SUPPLY USING FUZZY LOGIC
}

\author{
Chidurala Srinivas \\ Professor, Mechanical Engineering Department \\ Vaageswari College of Engineering \\ Karimnagar, Telangana State, INDIA
}

\begin{abstract}
In this paper, we have analyzed a serial operational level Supply Chain Performance like fill rate, Build-To - Stock etc., under an uncertainty environment for the optimality using a stochastic customer order. It evaluates minimum cost in supply chain, simultaneously optimize quantitative decision variables and illustrates the significance of capacity variable. Numerical examples are presented to illustrate the benefit of the proposed strategies and the effects of changes on the cost and parameters are studied.
\end{abstract}

\section{Notations used}

$\begin{array}{ll}\mu & : \text { Fuzzy membership function } \\ \mathrm{Q}_{\mathrm{c}} & \text { : Customer order quantity } \\ \mathrm{Q}_{\mathrm{w}} & \text { : Ware house inventory } \\ \text { BTS } & : \text { Build }- \text { To }- \text { Stock } \\ \mathrm{P}_{\mathrm{i}} & \text { : Product, } \mathrm{i}=1 \text { to } \mathrm{n} \\ \mathrm{rmi} & : \text { raw material inventorv }\end{array}$

$\begin{array}{ll}\mathrm{Pr}_{\mathrm{i}} & : \text { Process } i \\ \mathrm{epi} & : \text { end-product inventory } \\ \mathrm{d}_{\mathrm{rc}} & : \text { Random Customer Demand } \\ \mathrm{d}_{\mathrm{i}} & : \text { Low Customer Demand } \\ \mathrm{d}_{\mathrm{h}} & : \text { High Customer Demand } \\ \mathrm{d}_{\mathrm{m}} & : \text { Medium Customer Demand }\end{array}$

Keywords: Supply Chain, Fill rate, Uncertainty, Fuzzy logic, Inventory

Cite this Article: Chidurala Srinivas, Supply Chain Modeling with Uncertainty in Demand and Supply Using Fuzzy Logic, International Journal of Advanced Research in Engineering and Technology, 10 (2), 2019, pp 333-340.

http://iaeme.com/Home/issue/IJARET?Volume $=10 \&$ Issue $=2$

\section{INTRODUCTION}

Supply Chain (SC) is generally viewed as a network of facilities that perform procurement of raw materials, processing them into end products, distribution and selling them to end customers. A supply chain consists of all stages involved, directly or indirectly in fulfilling a customer request which includes manufacturers, suppliers, logistic providers, warehouse, retailers and finally the end customer. Each stage of supply chain performs different processes and shares the information with other stages of SC. Inventories may exist in the network of SC due to uncertainty in Business Environment. In the review on safety stock determination pointed out that there are two kinds of uncertainties in inventory control: Demand side uncertainty and Supply side uncertainty [1]. However most inventory models deal with the Demand side 
uncertainty and research on supply side uncertainty is relatively scant. In a typical two-echelon SC the inventories have been considered with either warehouse or supplier. Treating uncertainty is an important issue in supply chain modeling. Two inherent sources of uncertainties in SC environment are identified as customer demand and supply of raw material. If the warehouse has less than the fuzzy customer order quantity i.e., $\mathrm{Q}_{\mathrm{w}}<\mathrm{Q}_{\mathrm{c}}$ units in stock, first the partial order of size will be supplied from the stock, then the unfilled customer ordered quantity $\left(\mathrm{Q}_{\mathrm{c}}-\mathrm{Q}_{\mathrm{w}}\right)$ will be backordered from the warehouse. The supply of raw material uncertainty is correlated accordingly. In this paper these two uncertainties are represented by fuzzy logic based on fuzzy arithmetic rules and interpreted using fuzzy modeling.

The performance of Supply Chain is evaluated and the competitive and cooperative selections of stock level policies were determined. The customer fill rate at each stage in the two-echelon Supply Chain with one supplier and $\mathrm{P}_{\mathrm{i}}$ products for minimum total inventory cost of the entire serial production Supply Chain is modeled. The performance measures can be classified in to two categories Qualitative (customer satisfaction, product quality) and Quantitative measures (inventory levels, resource utilization, fill rate, total cost). The fill rate is the percentage of customer end product order quantity full filed by the ware house immediately from the stock on hand. Customer service level is a function of performance indices like the order fill rate, which is the fraction of customer demands that are met from the stock at ware house. [2]

\section{FUZZY LOGIC MODELING}

Fuzzy set theory provides a means for representing uncertainties [3]. Past few years have witnessed a rapid growth in the number and variety of applications of fuzzy logic. A fuzzy set contain the elements that have varying degree of membership in the set is measure as a function that attempts to describe vagueness and ambiguity [2]. This function maps elements of a fuzzy set $(\tilde{A})$ to a real numbered value on the interval 0 to 1 . If an elements in the universe, say $x$ is a member of fuzzy set $\tilde{A}$, then this mapping is given by $\mu_{\tilde{A}}(\mathrm{x}) \varepsilon[0,1]$. The feature of fuzzy logic modeling is shown in Figure 1. Fuzzy inference is a method that interprets the values in the input vector and, based on some set of rules, assigns values to the output vector. Fuzzification is the process of making a crisp quantity fuzzy. They carry considerable uncertainty. If the form of uncertainty happens to arise because of imprecision, ambiguity, or vagueness, then the variable is probably fuzzy and can be represented by a membership function. This is a intuitive type, which involves contextual and semantic knowledge about an issue. The combination of fuzzy conditions is determined by the minimum or average membership functions of the element of each proposition to the concerned fuzzy set. When fuzzy conditions of all rules have been quantified, fuzzy conclusions can be calculated. A key difference between crisp and fuzzy sets is that a crisp set has a unique membership function, whereas a fuzzy set can have an infinite number of membership functions to represent it. Fuzzy sets are always functions, which map a universe of objects. The function that maps onto $[0,1]$ is a fuzzy set. Defuzzification is the conversion of a fuzzy quantity to a precise quantity, just as fuzzification is the conversion of a precise quantity to a fuzzy quantity. The output of a fuzzy process can be the logical union of two or more fuzzy membership functions defined on the universe of discourse of the output variable. The defuzzification is used to convert the fuzzy set to a crisp value. Different methods are used for defuzzification, among which the center of the gravity is the most prevalent and physically appealing of all the defuzzification methods [1]. 


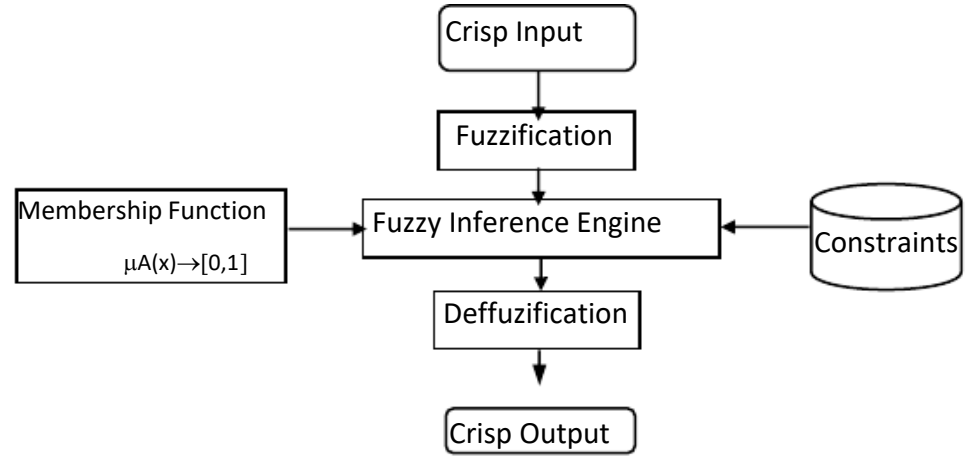

Figure 1 Fuzzy Logic Modeling

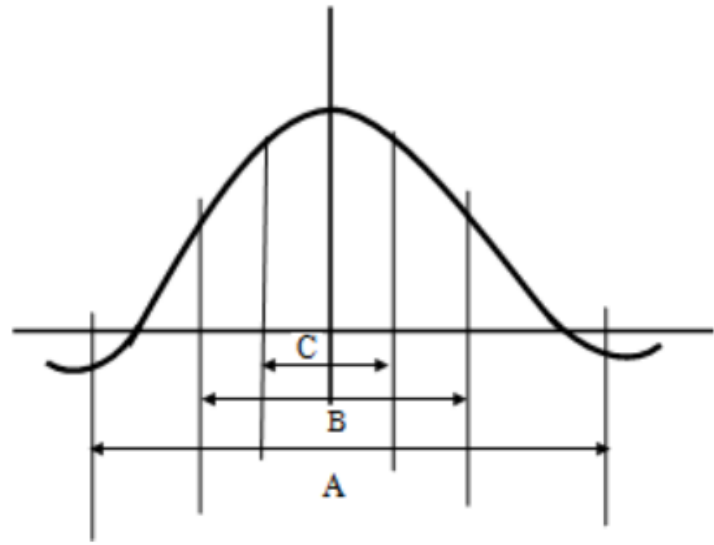

Figure 2 Customer service for different uncertainties

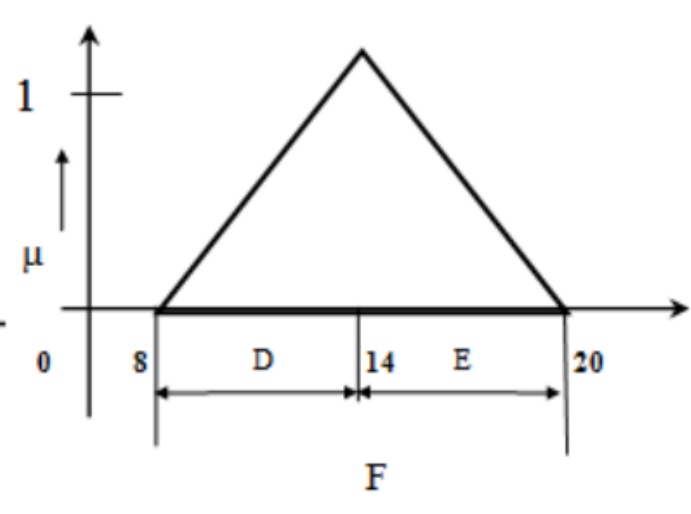

Figure 3 Raw material Inventory
A : Low degree uncertainty
$68.2 \%$
B : Medium degree uncertainty $95.45 \%$
C : high degree uncertainty $99.73 \%$
D : Reliable Supplier
E : Un-Reliable Supplier
F : Raw Material Inventory Range

\section{CASE STUDY}

In order to examine SC behavior in an uncertain environment, the authors have performed a simulation study and the flow chart of the program is given in Figure 5. The customer demand and external supplier reliability are specified by possibility distributions and transforms into the corresponding probability distribution and the corresponding datum described by a linguistic term which is based on a random number. The performance calculations have been done at operational level of supply chain network with BTS (Build-To-Stock) system of partial inventory at warehouse and BTO (Build-To-Order) system of partial back order[6] Some work has been done at multi-echelon serial raw material inventory/distribution systems to observe the performance variations of the system for different variables (Figure 4). It is found that the most of the cases the customer uncertainty demand have found an inevitable which causes to make some inventory levels at the ware house. The raw material requirement (external supplier) for the random backorders (unfilled customer demand) will be placed by the warehouse to the production facility which intern will be informed to the external supplier. After each replenishment of the customer order, the warehouse updates the inventory status every times. For the various iterations of analysis we have taken some of the input data (fuzzy sets) from the work done by the Dobrila Petrovic[1,4,5]. 


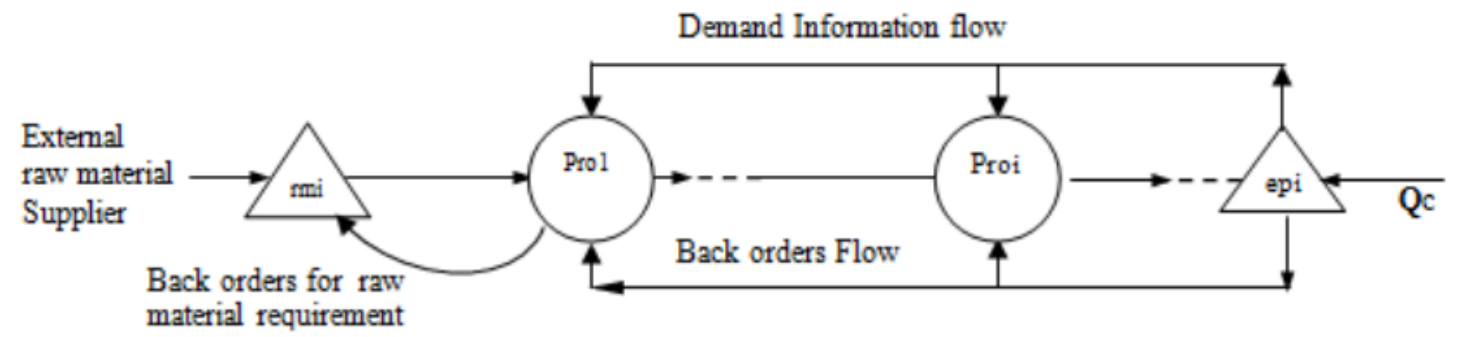

Figure 4 Schematic view of a serial supply chain

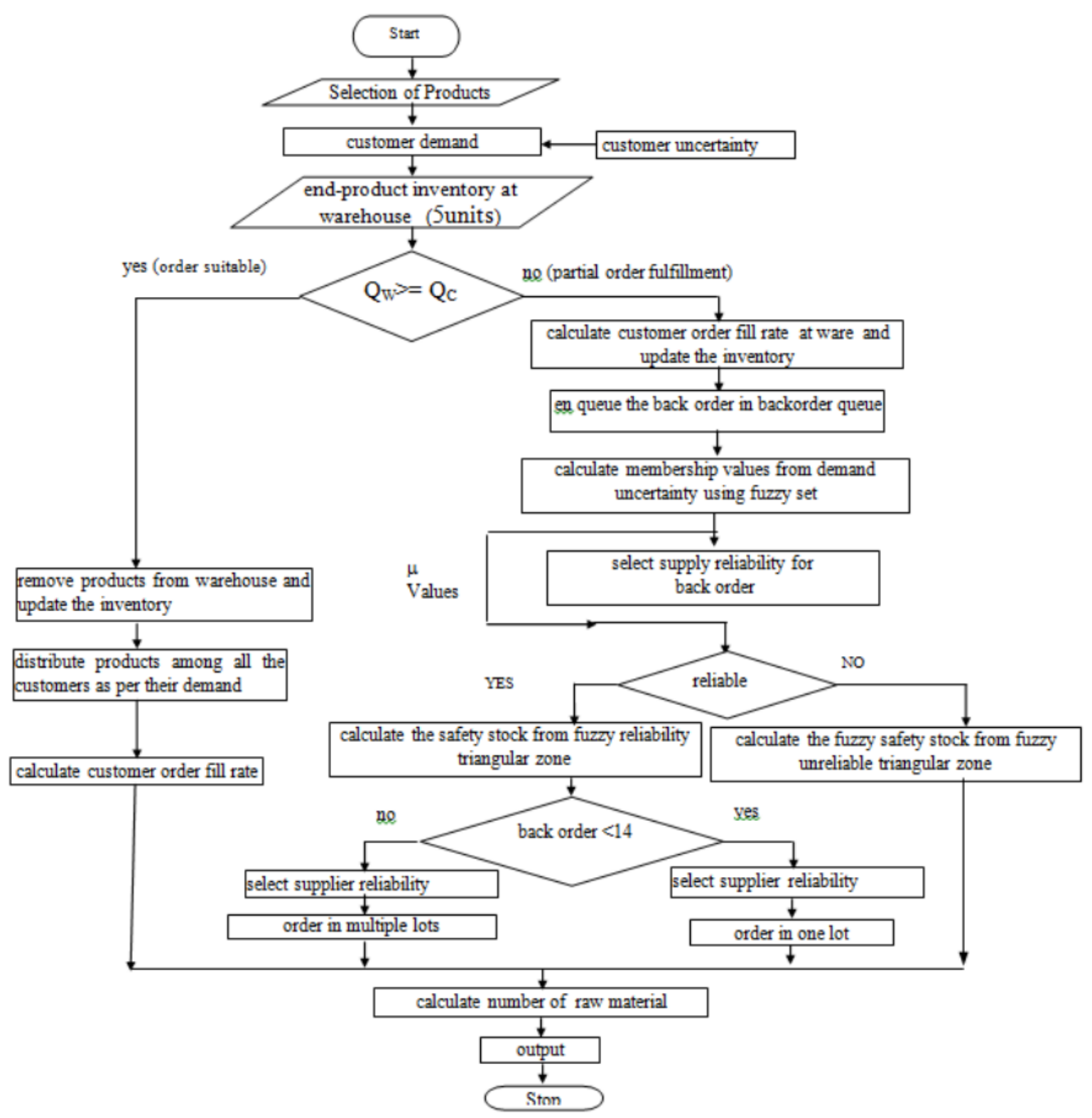

Figure 5 Case study modeling

\subsection{Iterations analysis}

Six test case iterations have been conducted with the customer uncertainty Iteration 1. High degree of customer demand uncertainty and reliable raw material supplier 
Iteration 2. Medium degree of customer demand uncertainty and reliable raw material supplier

Iteration 3. Low degree of customer demand uncertainty and reliable raw material supplier

Iteration 4. High degree of customer demand uncertainty and Un-reliable raw material supplier.

Iteration 5. Medium degree of customer demand uncertainty and Un-reliable raw material supplier.

Iteration 6. Low degree of customer demand uncertainty and Un-reliable raw material supplier.

High degree customer uncertainty: The customer demand in each time can have 13 possible integer values in the range from 4 to 16 and it is modeled by a discretized time stationary fuzzy set.

$\mathrm{D}=\{0.25 / 4,0.25 / 5,0.25 / 6,0.25 / 7,0.5 / 8,0.75 / 9,1 / 10,0.75 / 11,0.5 / 12,0.25 / 13,0.25 / 14,0.25 / 15$, $0.25 / 16\}$

Medium degree of uncertainty: Customer demand in this can have 7 possible integer values in the range from 7 to 13 and it is modeled by a discretized time stationary fuzzy set.

$\mathrm{D}=\{0.25 / 7,0.5 / 8,0.75 / 9,1 / 10,0.75 / 11,0.5 / 12,0.25 / 13\}$

Low degree of uncertainty: Customer demand in each time can have 3 possible integer values in the range from 9 to 11 and it is modeled by a discretized time stationary fuzzy set.

$\mathrm{D}=\{0.75 / 9,1 / 10,0.75 / 11\}$

The random customer demand uncertainty $d_{r c}$ will be in the range of $d_{r c}>d_{1}, d_{r c}<d_{u} \quad\left(d_{1}=4\right.$, $\mathrm{d}_{\mathrm{u}}=16$ ) Supply of raw material delivery along the Supply Chain may be unreliable in the sense that part of the quantity ordered may only be received. The reasons for that might be of different nature, in all the means the supplier reliability can be divided using imprecise linguistic terms, such as reliable or unreliable raw material supplier which can be determined by discretized time stationary fuzzy set (Figure 3).

$\mathrm{R}=\{0.25 / 8,0.5 / 10,0.75 / 12,1 / 14\}$

For reliable fuzzy raw material can be determined by discretized time stationary fuzzy set,

$\mathrm{R}=\{0.25 / 20,0.5 / 18,0.75 / 16,1.0 / 14\}$

Type of uncertainty

High degree of uncertainty

Moderate degree of uncertainty

Low degree of uncertainty

$\begin{array}{ll}\mathrm{n} & \sigma \\ 0.48 & 0.1 \\ 1.9072 & 0.09 \\ 3.7 & 0.08\end{array}$

Number of raw material supplied $=$ average fuzzy inventory $(\mathrm{x})+\mathrm{n}^{*} \sigma$

Fill Rate : Inventory at warehouse/Demand

Build-To-Stock : Inventory at Warehouse + Raw Material Inventory - Demand

Total Cost $\quad$ : Raw Material Cost + Manufacturing Cost + Inventory Carrying Cost

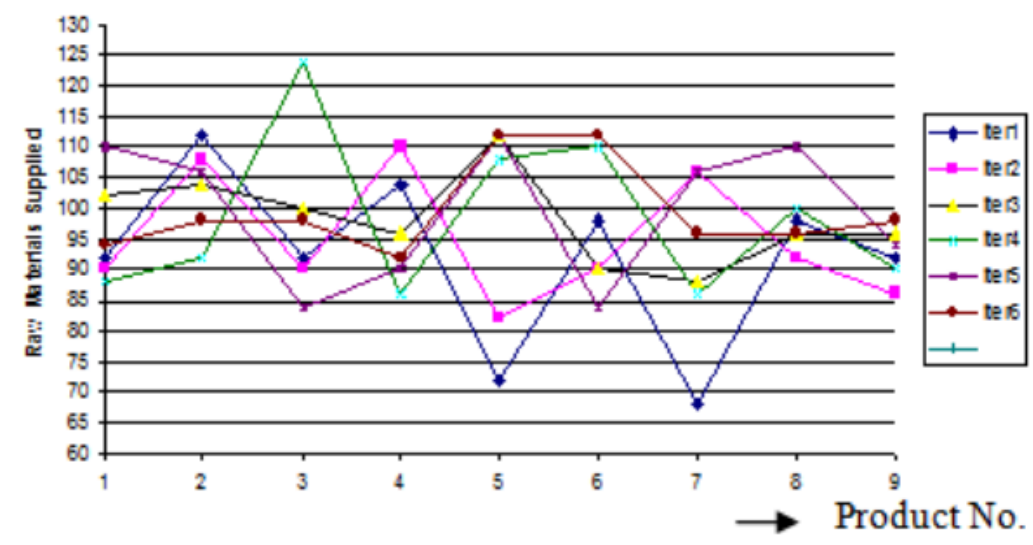

Figure 6 Raw Materials used for each Production Iteration 


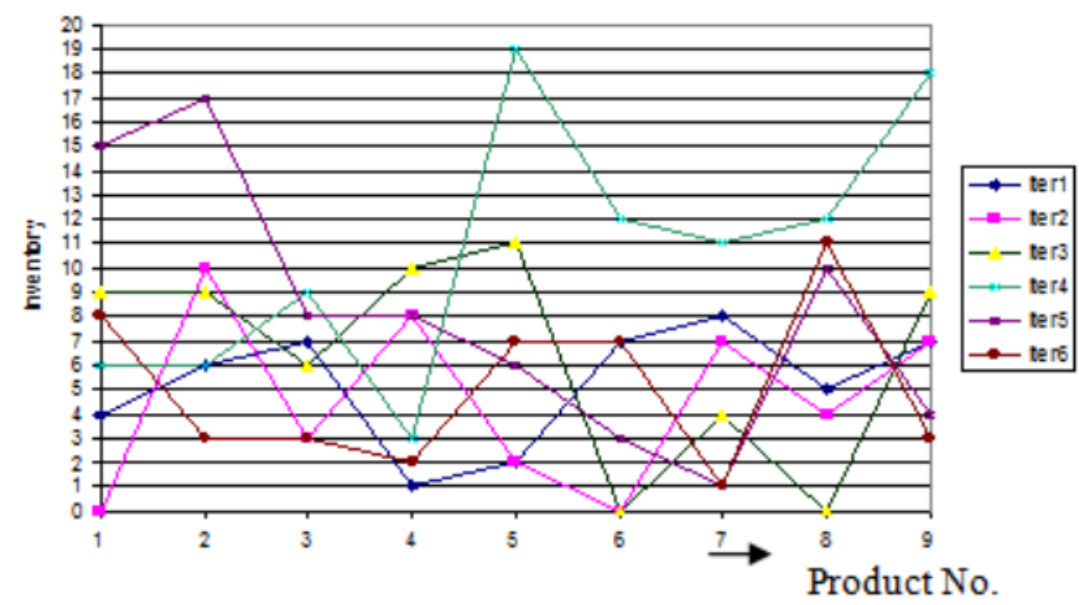

Figure 7 Inventories at warehouse for each product after the end of each Iteration

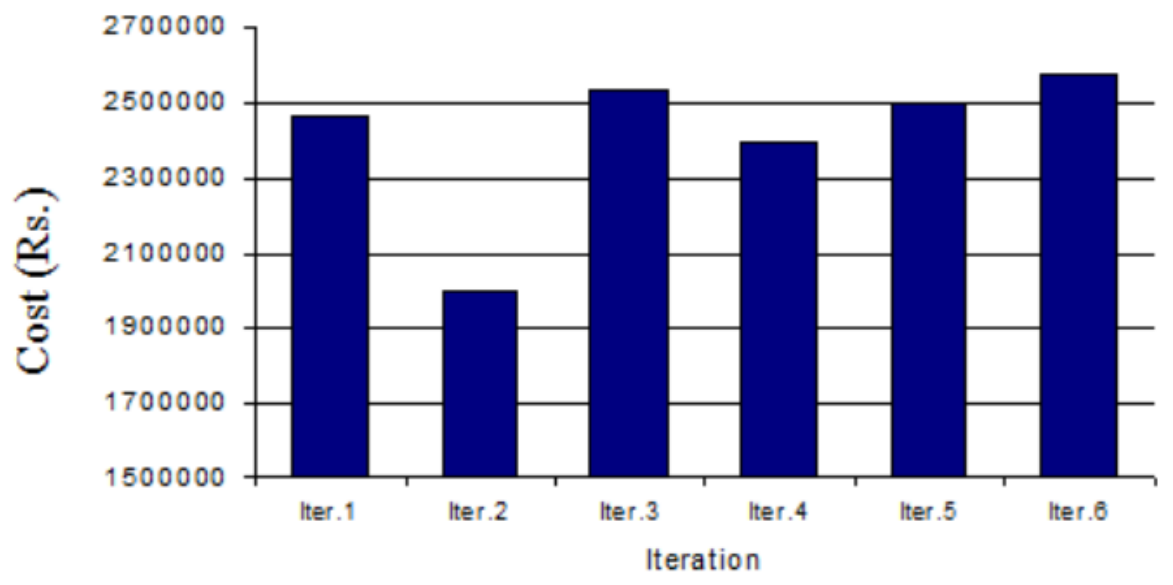

Figure 8 Total Cost in each Iteration

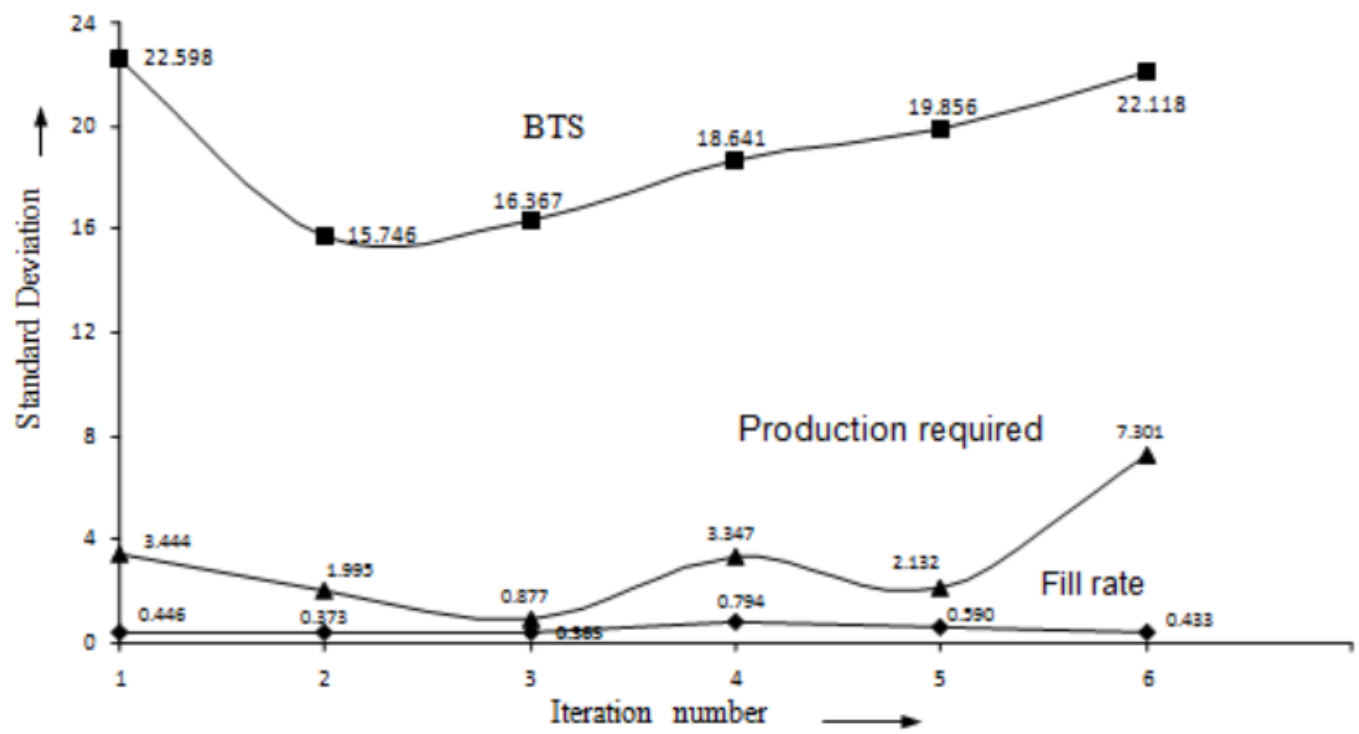

Figure 9 Standard Deviation Graph for Input (Production Required) Vs Output (BTS and Fill Rate) 


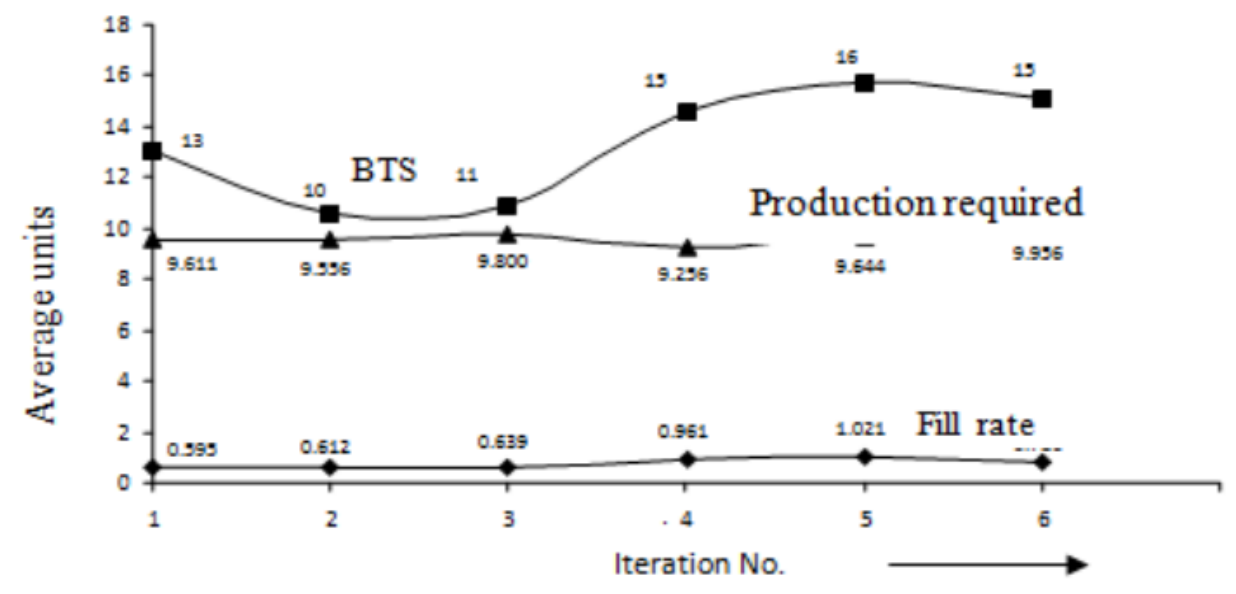

Figure 10 Input variables (Production Required) Vs Output variables (BTS and Fill Rate)

\section{CONCLUSIONS AND FUTURE WORK}

It has shown the uncertain customer demand and supply delivery along the SC have great impact on SC behavior. The average production requirement (all iterations) is in the range of 9-10(Input variable) and the out put variables like Fill-Rate and Built-To-Stock are in the range of 10-16(appr.) and 0.6-1.0(appr.) respectively. The output results shows that the fill rate is sensitive to customer uncertainty demand and supplier reliability (Figure 10). Iteration No. 2 gives less total cost (average of all the products) due to its nature of characteristics (medium degree of customer demand uncertainty and reliable raw material supply) where as for unreliable raw material supplier the total cost increases with decrease in uncertainty (Figure 8) and it is found that the Standard deviation for the entire Iteration No. 2 the Build-To-Stock \& Fill Rate is very less and more over it is towards downward trend of BTS (Figure 9). With random input variables lowest end-product stock levels reached before replenishments during the simulated time horizon in iteration 1 for cycles $1,2,3,6$ are $-5,-3,-2,-4$ and for iteration 4 for cycles 1, 4 are $-5,-5$ respectively. The negative stock levels denote the existence of shortage i.e., in all these cases, the inventory levels before replenishments are Zero/Negative, indicating insufficient order-up-to levels. For other stocks it is observed that the stock levels amplified along the chain, starting from the raw material inventory towards the end product inventory. The raw material being used in all iteration cycle varies between 82-124 (97 avg.) Where as there is always some items existing most of the times at warehouse for each product after the end of each iteration and varies from 0 to 19 (7 avg.) with the customer average order range of 9-10(avg.). There is lot of scope for improving with object library and can be added wider spectrum classes as generic reusable classes for the backorders.

\section{REFERENCES}

[1] Dobrila Petrovic, Rajat Roy, Radivoj Petrovic. "Modeling and Simulation of a Supply Chain in an Uncertain Environment" European Journal of Operations Research, vol.109, pp.299-309. (1998)

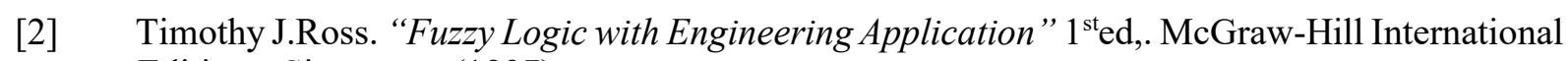
Editions, Singapore. (1997)

[3] Zadeh, L. A., Outline of a new approach to the analysis of complex systems and decision processes, IEEE Trans. SMC SMC-3, 98. (1973) 
[4] Dobrila Petrovic, Rajat Roy, Radivoj Petrovic,. "Supply Chain modeling using Fuzzy sets", International Journal of Production Economics, vol.59 pp.443-453. (1999)

[5] Dobrila Petrovic, Simulation of Supply Chain behavior and Performance in an Uncertain environment", International Journal of Production Economics, vol.71 pp .429-438. (2001)

[6] Jeremy F.Shapiro, "Modeling the Supply Chain", First Reprint, Thomson Asia pte. Ltd., Singapore. (2002)

[7] Ch. Srinivas, Decisions in a Supply Chain Modeling for Comparative Evaluation Strategies in Multiple Buyers, International Journal of Mechanical Engineering and Technology (IJMET), Volume 5, Issue 10, pp. 86-93, October (2014)

[8] Jamshed Siddiqui, An Exploration of Total Quality Management and Supply Chain Management Enablers, International Journal of Computer Engineering and Technology (IJCET), Volume 4, Issue 6, pp. 212-218, November - December (2013)

[9] Atul K. Shrivastava, Dr. S.K. Kumara Swamy, Anil Midathada and Uday K. Ravella Analysis of The Barriers for Implementing Green Supply Chain Management (GSCM) Practices on Organic Irrigation: An Analytical Hierarchy Process (AHP) Approach. International Journal of Mechanical Engineering and Technology, 8(7), pp. 1465-1473, 2017.

[10] Atul K. Shrivastava, Satyam Kumar, Dr. S.K. Kumara Swamy, Anil Midathada and Uday K. Ravella Analysis of The Barriers for Implementing Green Supply Chain Management (GSCM) Practices on Organic Irrigation: An Interpretive Structural Modelling (ISM) Approach. International Journal of Mechanical Engineering and Technology, 8(7), pp. 1446-1456, 2017

[11] Jeetesh Singh and Dr. J. Raghuram. Evolution of Supply Chain Management with Emerging Technologies. International Journal of Mechanical Engineering and Technology, 8(1), pp. 235240, 2017

[12] Ajay Verma and Dr. Anshul Gangele, Green Supply Chain Management Empirical Study for "Closing the Loop", International Journal of Production Technology and Management (IJPTM), Volume 3, Issue 1, pp. 78-94, January-December (2012).

[13] Dr. R. Prabusankar, Impact of Supply Chain Management Practices on Competitive Advantage of Small Manufacturing Firms in Coimbatore District, International Journal of Mechanical Engineering and Technology 8(10), pp. 836-843, 2017

[14] Ajay Verma and Dr. Anshul Gangele, Investigation Thought Decision-Making Trial and Evaluation Laboratory (Dematel) in Green Supply Chain Management Include Reducing and Recycling Pharmaceutical Waste for a Pharmaceutical Manufacturer in India, International Journal of Industrial Engineering Research and Development (IJIERD), Volume 3, Issue 2, pp. 58-73, July-December (2012)

[15] Hena Imtiyaz and Peeyush Soni, Marketing Supply Chain Management of Brinjal: Challenges and Opportunities, International Journal of Management (IJM), Volume 5, Issue 2, pp. 116-125, February (2014) 